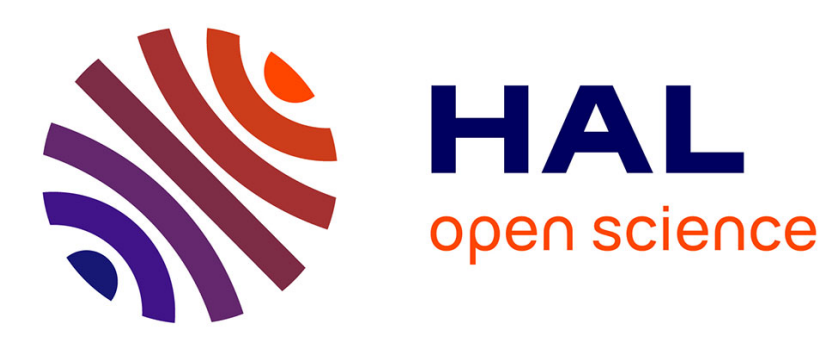

\title{
Interpretation of STM images: copper-phthalocyanine on copper
}

P. Sautet, Christian Joachim

\section{To cite this version:}

P. Sautet, Christian Joachim. Interpretation of STM images: copper-phthalocyanine on copper. Surface Science: A Journal Devoted to the Physics and Chemistry of Interfaces, 1992, 271 (3), pp.387-394. 10.1016/0039-6028(92)90902-I . hal-00006288

\section{HAL Id: hal-00006288 \\ https://hal.science/hal-00006288}

Submitted on 31 May 2021

HAL is a multi-disciplinary open access archive for the deposit and dissemination of scientific research documents, whether they are published or not. The documents may come from teaching and research institutions in France or abroad, or from public or private research centers.
L'archive ouverte pluridisciplinaire HAL, est destinée au dépôt et à la diffusion de documents scientifiques de niveau recherche, publiés ou non, émanant des établissements d'enseignement et de recherche français ou étrangers, des laboratoires publics ou privés.

\section{(c)(1)}

Distributed under a Creative Commons Attribution| 4.0 International License 


\title{
Interpretation of STM images: copper-phthalocyanine on copper
}

\author{
P. Sautet
}

Laboratoire de Chimie Théorique, ENS, 46, allée d'Italie, 69364 Lyon Cedex 07, France

ane'

Institut de Recherche sur la Catalyse, 2, avenue A. Einstein, 69626 Villeurbanne Cedex, France

and

C. Joachim

Molecular Electronic Group, 29, rue Jeanne Mariig, B.P. 4347, 31055 Toulouse Cedex, France

An interpretation of the constant-current STM image of a Cu-phthalocyanine molecule adsorbed on a Cu(100) surface is proposea based on an extension of the elastic scattering quantum chemistry (ESQC) technique to simulate STM images. After a brief description of the metinod, the dependence of the $\mathrm{Cu}$-phthalocyanine image on the tip structure and the Fermi level is presented. The way in which atom positions of an adsorbate can be extracted from an experimental STM image using the STM-ESQC technique is also discussed.

\section{Introduction}

Imaging a molecule in real space is an elegant way to get to know its exact structure, i.s., its size, shapc and the nature of its bonds [1]. Thinking about the field emission microscopy (FEM) activities, we remember that the resolution of the molecular skeleton was poor but molecules like phthalocyanine and flaventrene were truly observed [2]. Nowadays, images of molecules like benzene [3], naphthalene [4], phthalocyanine [5] or alkylcyanobiphenyl [6] are obtained using the scanning tunneling microscope (STM). But like in the early days of FEM, image mechanisms and contrast interpretation of the STM images are still a problem. Certainly, the STM image of a molecular adsorbate does not correspond to a simple van der Waals sphere mociel of the molecule because the tip shape convolutes with the adsorbate shape. Therefore, a first tentative explanation was to interpret the contrast by the definition of a local effective tunnel barrier at each $(x, y)$ STM tip position [7]. This leads to the evaluation of a local $(x, y)$-dependent pseudowork-function which depends on the imaged chemical species and on the chosen substrate. However, this interpretation does not produce more insight on the involvement of each molecular orbital of the adsorbate in the local contrast.

Next come the molecular orbital calculations on the isolated molecile, with the aim of determining the electron density map of certain crucial molecular orbitals of the ausorbate such as the lowest unoccupied (LUM )) ) or highest occupied (HOMO) molecular orbitais [5,6]. Although the symmetry of the experimental adsorbate print in the image is generally the same as the one given by these maps (as it was for FEM images), this print does no* fit in details the HOMO nor the LUMO electron density maps. More sophisticated is the use of the Tersoff-Hamann formula to make the connection between such calcula- 
tions and the tunneling current intensity [8]. However, it is known that the Bardeen approximation [y] ieacing io inc Torsoff-Hainann formula [8] is not applicable when the tip is close to the substrate or significantly coupled to the surface via an adsorbate [10]. Moreover, in a large majority of these calculations, nor the tip apex nor the surface are connected to an electron reservoir [11]. In this case, only the tip-apex-tosurface electronic coupling via the adsorbate can be calculated [19]. This electronic coupling is only indirectly related to the intensity of the tunneling current calculated when the tip apex and the surface are both connected to their respective bulk [13].

What we mean by interpreting an STM image can be separated in 3 parts: (a) to find on the experimental image the position of the atoms in the adsorbed molecule, (b) to understand why atoms in the molecule (or parts of the molecule) appear like holes or bumps in the image and, finally, (c) to evaluate tip-adsorbate deformations due to mechanical or electrostatic interactions between the tip and the adsorbate. Step (a) is generally not tackled by calculation. Often the atoms are positioned by hand on the image with the help of some symmetry (or characteristic shape) of the molecule. Step (b) has first been discussed by Lang [14] for isolated atoms. But his discussion was never extended to molecules where bonding and electron delocalization are important factors. For (c), structural deformations are small for "thin" molecules or for molecules lying flat on the surface. Therefore, they are not considered in the following. They might be important in the STM imaging of a thick organic morolayer [15] or of DNA [16]. Notice that when the adsorbates are unpinned on the surface, an important point to understand is also the mechanics of the tip-adsorbate push. Together with the tunneling current calculation, this requires an energy minimization of the tip-adsorbate-surface atomic structure to get the most stable one at each tip position. We have prefered in the following to consider a presupposed relaxed geometry for the adsorbate.

We present here our interpretations of the $\mathrm{Cu}$-phtaluryanine STM !mages based on the STM extension of our ESQC (elastic scattering quantum chemical) technique to calculate the intensity of a tunneling current [17]. This extension has already been shortly described [18] and details of calculations will be presented soon in a more technical paper [19]. Therefore, we prefer here to focus more on the interpretations of images than on the theory. Let us unly recall the principal steps of our model.

In the tunneling mode, the tip is very c ose (5 to $7 \AA$ ) to the substrate. Then, the system to consider cannot be the free adsorbate alone. It must be a supermolecule made of the tip apex, the adsorbate, and the substrate surface $[12,14]$. The electronic structure of this supermolecule is $(x, y, z)$-dependent with $(x, y, z)$ the coordinates of the STM tip apex relative to the substrate. Moreover, this supermolecule is strongly interacting with the tip wire and with the bulk of the substrate. In this case, for an electron coming from the tip wire (or frcm the bulk of the substrate), the supermolecule is a defect compared to the ordered electronir. structure met by the electrons in the tip and in the substrate. Scattering on this defect is mainly elastic due to the small size of the STM tunneling junction. At a low bias voliage $V$, the tunneling current intensity $I(x, y, z)$ through such a $(x, y, z)$-dependent defect is given by the generalized Landauer formula [20]. This formula relates the conductance $I(x, y, z) V^{-1}$ of the defect to the trace of the squared transmission matrix through this defect taken at the bulk Fermi level [21]. This matrix is obtained following our ESQC procedure [22] and using extended Hückel molecular orbital (EHMO) calculations [23]. Therefore, we calculate the tunneling current intensity with the tip apex and surface connected to electron reservoirs but without the standard Fermi golden rule (or Bardeen) approximation [9]. Notice that the effective electronic coupling between the tip apex and the surface through the adsorbate cari aiso be extracted from our calculations [22]. Moreover, this effective coupling can serve to define at each $(x, y, z)$ tip position an effective tunnelin, potential barrier as proposed in ref. [7]. The barrier height so evaluated can be plotted as a funcion of $(x, y, \ldots)$. ' 
tial is, e.g., the image force effect since we are dealing by hypothesis with a very low bias voitage. In extending ESQC to STM, we have considered that the calculation of STM images can be made equivalent to the now standard evaluation of the conductance of a disordered material (a defect) connected to ideal conducting leads. The only difference is that the electronic structure of the defect changes under STM tip scanning.

The paper is organized as follows. In section 2 the conditions for the calculation of the constant-current images obtained for the $\mathrm{Cu}$ phthalocyanine on $\mathrm{Cu}(100)$ are detailed. In section 3, the shape of the calculated STM images is discussed together with the possibility to extract the atomic structure of an adsorbate from its STM experimental image using its calculated image. In section 4 , the apparent corrugation on the STM print of the $\mathrm{Cu}$-phthalocyanine is interpreted using the electronic structure and electronic level position of the adsorbate $\mathrm{Cu}$ phthalocyanine compared to the copper bulk Fermi level.

\section{Calculation of the $\mathrm{Cu}$-phthalocyanine STM image in the constant-current mode}

An isolated $\mathrm{Cu}$-phthalocyanine molecule can be imaged if one searches for isolated ones around deposited aggregates or if these molecules are deposited on a fresh surface by the tip. Following this last recipe, STM images of an isolated $\mathrm{Cu}-$ phthalocyanine on a GaAs surface have been obtained first by Gimzewski and co-workers [24] followed by Möller and co-workers [25]. Higherresolution images of isolated $\mathrm{Cu}$-phthalocyanines have been obtained by Lippel and co-workers [5] on a $\mathrm{Cu}(100)$ surface. In this case, the tunneling current intensity was between 2 and $6 \mathrm{nA}$ for a tip-to-substrate bias voltage between -70 and $-390 \mathrm{mV}$. The maximum corrugation found between the top of the $\mathrm{Cu}$-phthalocyanine crown and the $\mathrm{Cu}(100)$ surface is $2.0 \AA$. Since atomic resolution was also reached on $\mathrm{Cu}(100)$ in this experiment besides the adsorbate, we chose to calculate these STM images of $\mathrm{Cu}$-phthalocyanine on $\mathrm{Cu}(100)$.

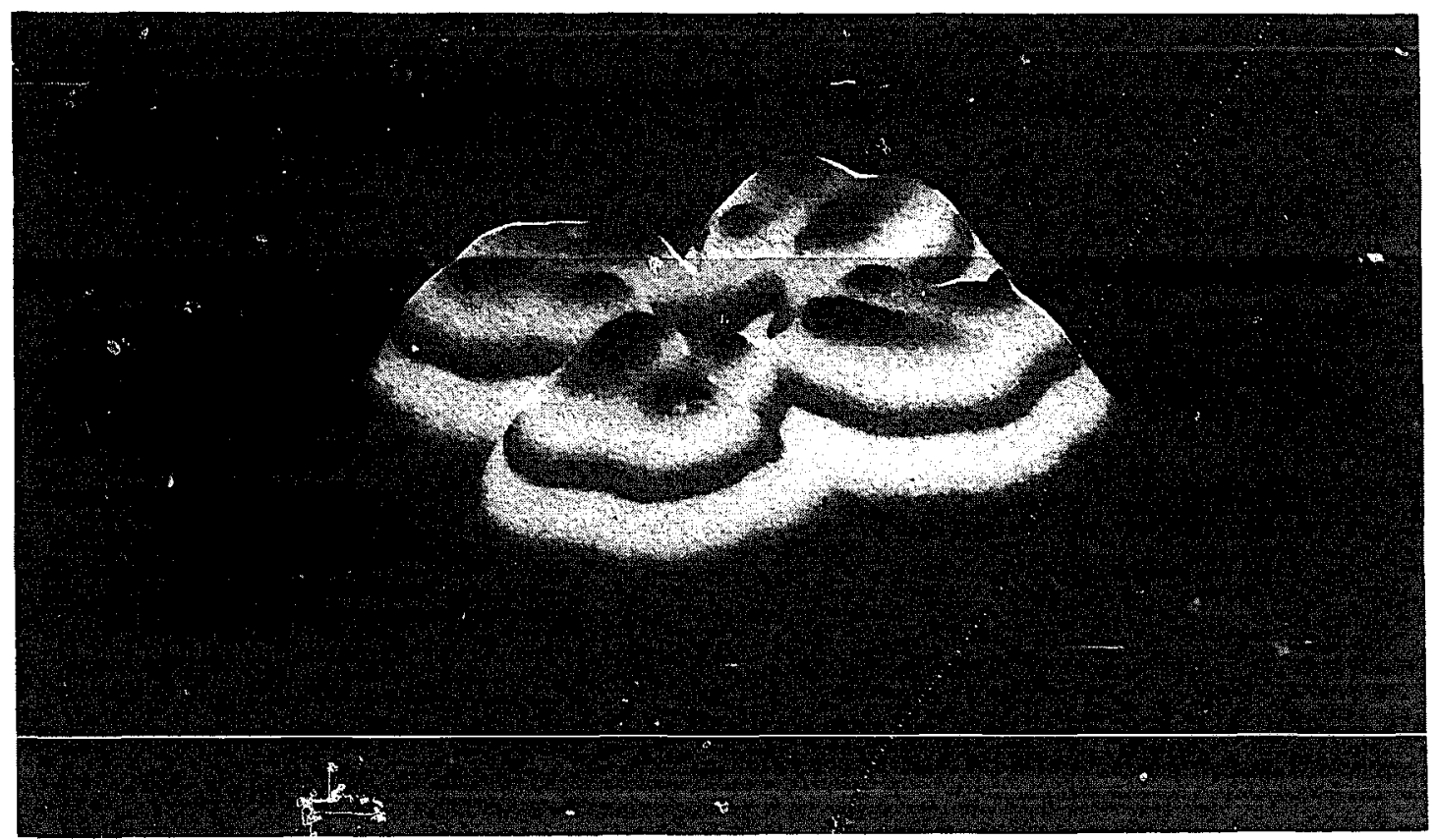

Fig. 1. STM constant-current calculated image of a $\mathrm{Cu}$-phtalocyanine on $\mathrm{Cu}(100)$. Tunneling current is $3 \mathrm{nA}$, tip bias voltage 50 $\mathrm{mV}, E_{\mathrm{f}}=-10.8 \mathrm{eV}$, image size $23 \times 23 \AA^{2}$ and $z$ linear scalc: $1.37 \AA$ from the copper surface to the top of the crown. The EHMO Hamiltonian of the tip-apex-molecule-copper-surface supermo ecule is built using the $4 \mathrm{~s}$ copper orbital for all the cnpper atoms in the supermolecule plus all the valence orbitals for the phtalocyanine organic skeleton. Cyclic boundary conditions are used for both the tip wire and the bulk substrate to get their metal bulk electronic band structure without lateral quantification effects. 
The Cu(100) surface is described by two layers of copper atoms each made of 64 atoms in an $8 \times 8$ square lattice with respect to the crystallographic distances. The bulk copper supporting the $\mathrm{Cu}(100)$ surface is made of a periodic repetition of these two layers which gives the bulk regular fcc copper structure. For the tip wire, the same unit cell as for the bulk substrate was chosen. The tip apex is made of a single copper atom bound to the tip wire surface. A larger tip apex, such as a 4-atom tetrahedron, can also be used. However, it was found unnecessary to use such a tip for a flat-lying molecule like the $\mathrm{Cu}$-phthalocyanine. For the position of the $\mathrm{Cu}$-phthalocyanine molecule's atoms, we have followed the geometry proposed by Somorjai and co-workers [26]. The molecule orientation on the $\mathrm{Cu}(100)$ face is only tentative [26] and must be confirmed by molecular mechanics calculation and by a comparison with the experimental images.

A first calculated constant-current image is presented in fig. 1 . The shape of the print created by the $\mathrm{Cu}$-phthalocyanine is in good agreement with published experimental images. The only problem is the apparent hole in the calculated image at the place of the central copper atom. This hole seems to be filled up in some of the experimental images. The image in fig. 1 is made of $69 \times 39$ pixels. Each pixel is obtained after an optimization ( 3 steps in average) of the $z$ tip altitude to get the set up current. In the scanning to get this image, the tip apex was so close to the $\mathrm{Cu}(100)$ surface in its parts free of molecules $(4.3$ $\AA$ in average) that the metallic corrugation is

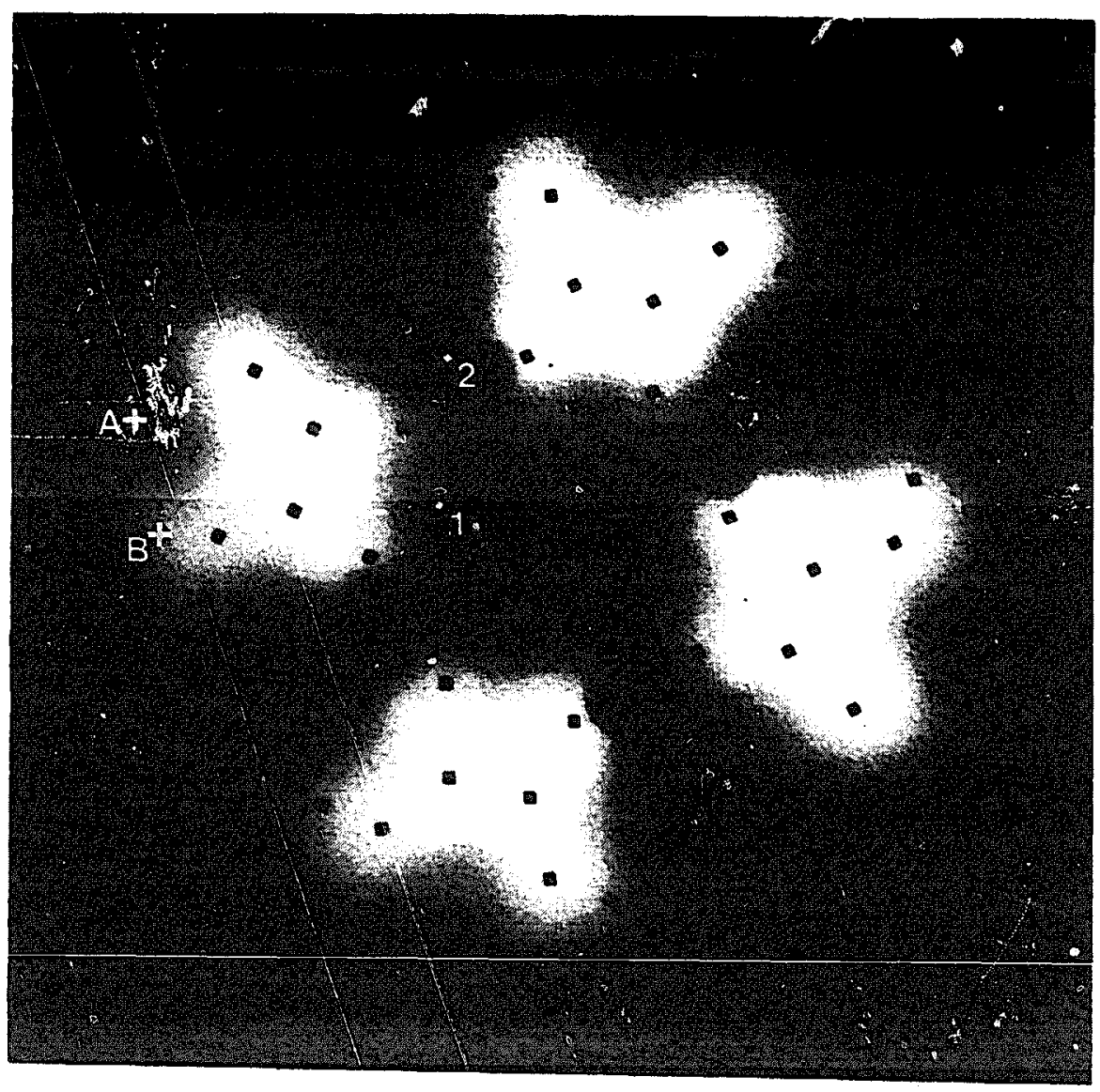

Fig. 2. Top-view constant-current calculated STM image with an optimized tip apex electronic structure description. Same scanning conditions as in fig. 1 with $E_{\mathrm{f}}=-10.3 \mathrm{eV}$ and with the image size restricted to $17.9 \times 17.9 \AA^{2}$. The $z$ linear scale is reduced to $1.08 \AA$ from black to white. The black squares on the image are the position of the $\mathrm{Cu}$-phtalocyanine atoms chosen for the calculation. The two types of nitrogen in $\mathrm{Cu}$-phtalocyanine are labeled with 1 and 2. 
clearly visible. This is due to our first choice of the atomic orbitals used to describe the tip apex electronic structure. For the calculated image in fig. 1, each copper atom is described by its $4 \mathrm{~s}$ Slater atomic orbital with its exponent and energy given by the standard EHMO parametrization [23]. Therefore, the extension of this type of orbital in space is not enough to ensure an order of magnitude variation of the tunneling current per $1 \AA$. In fact, the logarithmic slope of the current as a function the tip apex altitude is more than twice this value, which explains the short average distance found on a naked $\mathrm{Cu}(100)$ surface. Standard EHMO paramoters and bond distances were also used to describe the $\mathrm{Cu}$ phthalocyanine electronic structure. The Fermi level chosen for this image is $E_{\mathrm{f}}=-10.8 \mathrm{eV}$. and was estimated on a bulk copper. Moreover, the tunneling current is calculated from the Landauer formula, i.e., at constant energy $E_{\mathrm{f}}$ for both sides of the junction. Let us recall that to calculate the tunneling current intensity, the multichannel transmission matrix $[20,21]$ is calculated from the EHMO Hamiltonian following our ESQC method [17,18]. The band structure of the bulik copper substrate and copper tip wire needed to calculate the scattering matrix is only described by its $4 \mathrm{~s}$ band in the present calculations. ' 'here is no difficulty to extend the band description to $p$ and $d$ bands. The computation time will then increase in consequence.

Fig. 2 presents an image obtained after the optimization of the electronic structure of the tip apex end atom. The criterion for this is to reproduce on free $\mathrm{Cu}(100)$ a good current-distance characteristic with a decrease in current of one order of magnitude per $1 \AA$ variation of the tip altitude. Compared to the scan in fig. 1, this does not lead to atomic resolution on the free $\mathrm{Cu}(100)$ since the tip is now scanning at an higher altitude (5.3 $\AA$ in average). For this image, the chemical description of the $\mathrm{Cu}$-phthalocyanine copper atom is also completed by its $3 \mathrm{~d}$ and $4 \mathrm{p}$ atomic orbitals. There are some changes in the bumps compared to the irnage in fig. 1 due to a shift in the bulk copper Fermi level $E_{\mathrm{f}}$ from -10.8 to $-10.3 \mathrm{eV}$. This shift was introduced to ensure that $E_{\mathrm{f}}$ wili be wel! positioned in the middle of the HOMO-LUMO $\mathrm{Cu}$-phthalocyanine gap. However, the copper atom in the $\mathrm{Cu}$-phthalccyanine center is not visible.

Aside from the optimization of its electronic structure, other optimizations of the tip, such as shape and pollution, may be important. In our method, the tip apex is built atom by atom. Therefore, one can change the tip shape for example from a tetrahedron to a trigonal pyramide. One is also free to put adsorbates on the tip and even replace metal tip atoms by others. But all these changes must be tried only when standard tip structures have failed to reproduce the experimental results. This was, for example, the case in interpreting the $2 \times 2$ sulfur on rhenium STM images [27]. To reproduce the experimental images, it was necessary to replace the tip apex end atom by a sulfur atom [27].

\section{Position of the atoms on the $\mathrm{Cu}$-phthalocyanine STM image}

As already noticed in the introduction, atoms are usually positioned by hand on the images with the help of symmetry. For the $\mathrm{Cu}$-phtalocyanine, the $\mathrm{C} 4$ symmetry is retained both on the calculated and on the experimental images. However, there is no atomic detail on the calculated image even if the number of calculated pixels is increased. Furthermore, as already noticed, the $\mathrm{Cu}$-phthalocyanine copper central atom does not show up in the center of the print. Some details are apparent on the crown. They are Fermilevel-dependent but do not correspond to any atomic position.

Moreover, the advantage of our calculation is that the exact position of each atom of the $\mathrm{Cu}-$ phthalocyanine skeleton is known because we have chosen them. The first important conclusion is that compared to these positions, the lateral extension of the $\overline{\mathrm{Cu}}$-phthaiocyanine print on the image in fig. 2 is larger (further than the hydrogen atoms) than expected from a LUMO or HOMO electron density map [5]. This is a general aspect of molecular adsorbate STM imaging: the overlap between the tip apex and adsorbate orbitals is not zero even at large lateral distances, 
compared to standard interatomic distances, between the two. This results in a non zero tunneling current when the tip is in the lateral neighbourhood of the adsorbate. This contributes to the apparent expansion of the adsorbate print in its STM image compared to the position of its atoms. Interferences between through-adsorbate and through-space tunneling processes participate to this effect which is orbital-symmetry- and tip-shape-depenaent. A local density-of-states calculation $[8,14,28]$ is not adapted to grasp this effect since the tip is considered far away from the surface. Moreover, in such calculations, the tip radius or the tip-substrate distance is generally optimized to fit the lateral size of the adsorbate print with the experimental image $[8,28]$.

To push further in the direction of the extraction of atomic positions, a fitting between the experimental and the calculated image is required. The displacement of any atom in the adsorbate skeleton has a signature on the STM print even if the consequence of this move is not the direct displacement of a bump on the image. More difficult in this perspective is the CPU time required for such calculations. To minimize the experimental-to-calculated STM image distance, one needs not only try a large number of possibilities for the atomic positions in the adsorbate. The tip structure must also be optimized. This can be done in principle with images of naked surfaces supposing that the tip structure does not change from the naked to the covered surface. But such a full optimization of the tip-adsorbate-surface supermolecular geometry appears to be still ahead due to the computation time required.

\section{Interpretation of the apparent corrugation on the image}

For a large adsorbate with many orbitals available near $E_{\mathrm{f}}$, the electron density map constructed from one of the orbitals in resonance with $E_{\mathrm{f}}$ cannot reproduce all the details of an experimental image. One needs to superpose at $E_{\mathrm{f}}$ all the through-bond and through-space contributions to the tunneling current [22]. They may come from orbit als out of resonance with $E_{\mathrm{f}}$. The reason is that the interaction between an orbital of the adsorbate and one of the tip is proportional only to the inverse energy difference between these two orbitals. For an orbital out of resonance, it is the tail of the resonance taken at $E_{\mathrm{f}}$ which must be considered [22,28]. The superposition of all these orbital contributions is not additive due to interference effects $[22,17]$. Therefore, even a superposition of the local density of states cannot reproduce the detailed corrugation. It is the specific position of the resonances and their mutual interferences which are responsible for the STM corrugation. This rules
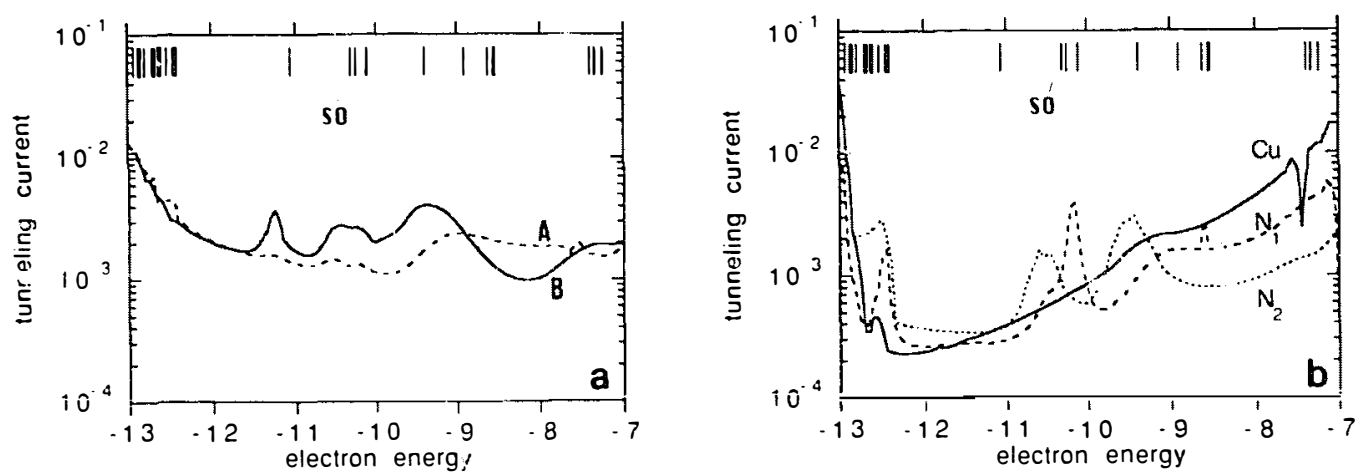

Fig. 3. Variation of the tunneling current as a function of the electron energy for a fixed $4.2 \AA z$ tip altitude from the $\mathrm{Cu}$-phtalocyanine. (a) Is for two different tip positions $\mathrm{A}$ and $\mathrm{B}$ specified by a star on the scan in fig. 2, and (b) is for the tip positioned on the nitrogen atom N1, N2 and on the central copper atom. The position of the $\mathrm{Cu}$-phtalocyanine molecular orbital energy levels are specified for the energy range chosen with SO the single and highest occupied orbital. The current intensity is in $\left(2 e^{2} h^{-1}\right) \mathrm{V}$. 
out the distinction of atoms in the STM image of a large adsorbate. Functional groups may not have the same signature if included in different adsorbates or if the substrate has changed. Moreover, the local-density-of-states approximation produces the overall shape of an adsorbate STM image since the $(x, y)$ variations of $I(x, y, z)$ depend on the spatial shape of the considered orbitals and therefore of the corresponding local density of states. But this is not enough to recognize an adsorbate without using symmetry or its characteristic shape.

On the scan in fig. 2, some nitrogens contribute a little to the corrugation and others are not visible. Moreover, the carbon skeleton produces $E_{\mathrm{f}}$-dependent bumps. To interprete the bumps and holes, fig. 3 presents the $E_{\mathrm{f}}$ variation of the tunneling current for different $(x, y)$ tip positions at a fixed $z$ altitude over the $\mathrm{Cu}-$ phthaiocyanine. The differences in corrugation in figs. 1, 2 and 4 between the tip positions A and B, defined in fig. 2 , are interpreted by looking at the molecular orbital resonance positions (fig. 3a). Between -12.0 and $-8.0 \mathrm{eV}$, there are 3 resonances at position $\mathrm{B}$, only a flat one at position $\mathrm{A}$ and each can be indexed to a $\mathrm{Cu}$-phthalocyanine molecular orbital. The change in current from $A$ to $B$ is controlled by symmetry matching between the tip and the $\mathrm{Cu}$-phthalocyanine orbitals. For example, for the tip at position $\mathrm{A}$, the resonance at $E=-9.0 \mathrm{eV}$ produces a bump (fig. 4) which disappears on the scans in figs. 1 and 2 . From fig. $3 \mathrm{~b}$, nitrogens $\mathrm{N} 1$ cannot produce bumps at the same $E_{\mathrm{f}}$ 2.s nitrogens $\mathrm{N} 2$, since the resonances for

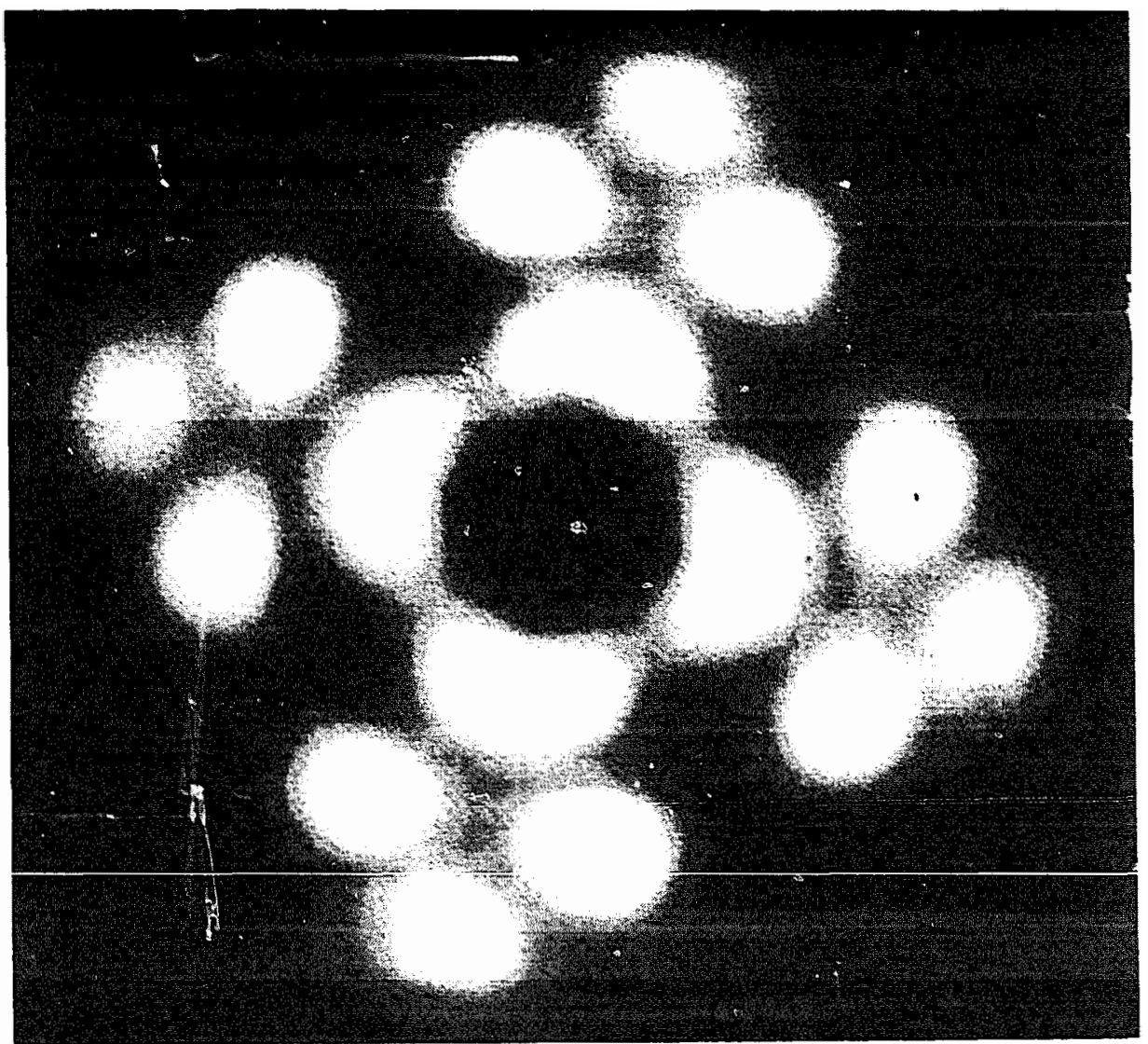

Fig. 4. Tep-view constant-current calculated STM image with the same conditions as in fig. 1 but with $E_{1}-8.8 \mathrm{i}$. Til - iilleat scale is $1.25 \AA$ from black to white. 
the tip positioned on N1 do not occur at the same encrgy as the $\mathrm{N} 2$ ones. A comparison between figs. $3 a$ and $3 b$ demonstrates that the current intensity is generally lo.ver on the N1 and N2 as on the carbon skeleton. The only exception is at $E=-10.2 \mathrm{eV}$ where the $\mathrm{N} 1$ resonances produce a higher tunneling current leading to a non zero corrugation on these nitrogens in some scans. Each of the corrugation details on the scans in figs. 1, 2 and 4 can be analyzed in this way.

\section{Conclusion}

Based on quantum chemistry calculations, simulations and shape predictions of the STM image of a molecular adsorbate of the size of the $\mathrm{Cu}-$ phthalocyanine are now available. Re ognition of a small adsorbate and tip structure detcrmination are in principle possible. By moving each atom step by step in calculating an image, the distance between experimental and calculated images can be minimized and the atomic structure of the adsorbate extracted from its expermental STM image. Our technique is in principle also applicable to predict STM images of larg? adsorbates like DNA. The computing time required is one limitation of such calculations. Others are the evaluation of electric and mechanical forces acting on the adsorbates during a scan. The mechanical deformation can in principle be handled using a molecular dynamic approach. But computing time is also a limitation. Electric field effects are more complex to deal with. Using an SCF procedure in calculating the tunneling current to take into account adsorbate polarization effects seems find the principle. But added to the calculation of mechanical effects, the calculation and interpretation of an STM image becomes a tremendous computing problem.

\section{Ackno:vledgements}

We thank A. Altibelli and J. Aussoleil from CEMES for their help in processing the images.

\section{References}

[1] C.A. Coulson, The Shape and Structure of Molecules (Clarendon Press, Oxford, 1978).

[2] A.J. Melmed and E.W. Müller, J. Chem. Phys. 29 (1958) 1037.

[3] H. Ohtani, R.J. Wilson, S. Chiang and C.M. Mate, Phys. Rev. Lett. 60 (1988) 2398.

[4] V.M. Hallmark, S. Chiang, J.K. Brown and Ch. Wöll, Phys. Rev. Lett. 66 (1991) 48.

[5] P.H. Lippel, R.J. Wilson, M.D. Miller, Ch. Wöll and S. Chiang, Phys. Rev. Lett. 62 (1989) 171.

[6] D.P.E. Smith, J.K.H. Hörber, G. Binnig and H. Nejoh, Nature 344 (1990) 641.

[7] J.K. Spong, H.A. Mizes, L.J. Lacomb Jr., M.M. Dovek, J.E. Frommer and J.S. Foster, Nature 338 (1989) 137.

[8] J. Tersoff and D.R. Hamann, Phys. Rev. B 31 (1985) 805.

[9] J. Bardeen, Phys. Rev. Lett. 6 (1961) 57.

[10] A.A. Lucas, Europhys. News 21 (1990) 63; C. Noguera, J. Je Phys. (Paris) 50 (1989) 2587.

[11] M. Tsukada, K. Kobayashi and S. Ohnishi, J. Vac. Sci. Technol. A8 (1989) 160.

[12] A. Farazdel and M. Dupuis, Phys. Rev. B 44 (1991) 3909.

[13] P. Sautet and C. Joachim, J. Phys. C 21 (1988) 3939.

[14] N. Lang, Phys. Rev. Lett. 56 (1986) 1164.

[15] B. Michel, G. Travaglini, H. Rohrer, C. Joachim and M. Amrein, Z. Phys. B 76 (1989) 99.

[16] S.M. Linsay, O.F. Sankey, Y. Li, C. Herbst and A. Rupprecht, J. Phys. Chem. 94 (1990) 4655.

[17] P. Sautet and C. Joachim, Chem. Phys. Lett. 153 (1988) 511.

[18] P. Sautet and C. Joachim, Chem. Phys. Lett. 185 (1991) 23.

[19] P. Sautet and C. Joachim, in preparation.

[20] A.D. Stone and A. Szafer, IBM J. Res. Dev. 32 (1988) 384.

[21] P.W. Anderson, D.j. Thouless, E. Abranams and D.S. Fisher, Phys. Rev. B 22 (1980) 3519.

[22] C. Joachim and P. Sautet, in: Scanning Tunneling Microscopy and Related Methods, Eds. R.J. Behm, N. Garcia and H. Röhrer (Kluwer, Dordrecht, 1590) p. 377.

[23] R. Hoffmann, J. Chem. Phys. 39 (1963) 1397.

[24] J.K. Gimzewski, E. Stoll and R.R. Schlittler, Surf. Sci 181 (1987) 267.

[25] R. Möller, R. Coenen, A. Esrlinger and B. Kaslouski, J. Vac. Sci. Technol. A 8 (1990) 659.

[26] J.C. Buchholz and G.A. Somorjai, J. Chem. Phys. 66 (1977) 573.

[27] J. Dunphy, D.F. Ogletree, M.B. Salmeron, P. Sautet, M.L. Bocquet and C. Joachim, Ultramicroscopy (1992), in press.

[28] D.M. Eigler, P.S. Weiss, E.K. Schweizer and N. Lang, Phys. Rev. Lett. 66 (1991) 1189. 